\title{
The different lives of galaxies at different environment density levels
}

\author{
Antti Tamm ${ }^{1}$, Lauri Juhan Liivamägi ${ }^{1}$ and Elmo Tempel ${ }^{1,2}$ \\ ${ }^{1}$ Tartu Observatory, Tõravere, 61602 Tartumaa, Estonia \\ email: antti.tamm@to.ee \\ ${ }^{2}$ National Institute of Chemical Physics and Biophysics, Rävala pst 10, 10143, Tallinn, Estonia
}

\begin{abstract}
We take a closer look at the dependence of the galactic colour histogram on the environment density using a volume-limited sample of SDSS galaxies. We find that the strongest changes with environment are taking place with spiral galaxies. In dense environment, discs become considerably redder, apparently due to the shortage of gas, and less concentrated. Contrary to expectation, the mean Sérsic index of luminous elliptical galaxies decreases in denser environments.
\end{abstract}

Keywords. galaxies: photometry - galaxies: structure - galaxies: spiral - galaxies: elliptical

\section{Introduction}

The dependence of galaxy properties on the environment has been known since the papers by Einasto et al. (1974), Oemler (1974), Davis \& Geller (1976), Dressler (1980), Postman \& Geller (1984). The most pronounced effect is the morphology-density relation, which states that the fraction of elliptical galaxies increases at the cost of spiral galaxies in cluster environment with respect to field environment. Exploiting data from extensive redshift surveys, more recent studies have revealed several additional effects of the environment. It has been shown that in addition to the local (i.e. group and cluster) neighbourhood, also the larger-scale environment density (e.g. void vs superclulster) has an impact on galaxy populations (Tempel et al. 2011, Lietzen et al. 2012, Einasto et al. 2014). For example, the luminosity function of elliptical galaxies depends on the largescale environment density, while spiral galaxies maintain their luminosity function shape.

The transformation of blue star-forming spiral galaxies into red and dead ellipticals and S0-s in dense environments apparently takes place via two major mechanisms: galaxygalaxy interactions and gas-gas interactions. While the former transforms the structure of galaxies via matter redistribution, the latter does not. Instead, removal of gas suppresses star formation, but has no immediate effect on the galactic structure. As a result, the bimodal colour distribution, corresponding to a bimodal star formation rate distribution, depends heavily of the environmental density (Kauffmann et al. 2004).

In this contribution we take a closer look at the impact of environmental desity on galaxy properties by investigating the colours and structural properties of galaxies within high- and low-density large-scale environments.

\section{Input}

The galaxy sample is based on a volume-limited sample of the Sloan Digital Sky Survey (SDSS; York et al. 2000) data release 10 (Ahn et al. 2014), taken from the galaxy group catalogue (Tempel et al. 2014). The whole sample consists of 105,041 galaxies 


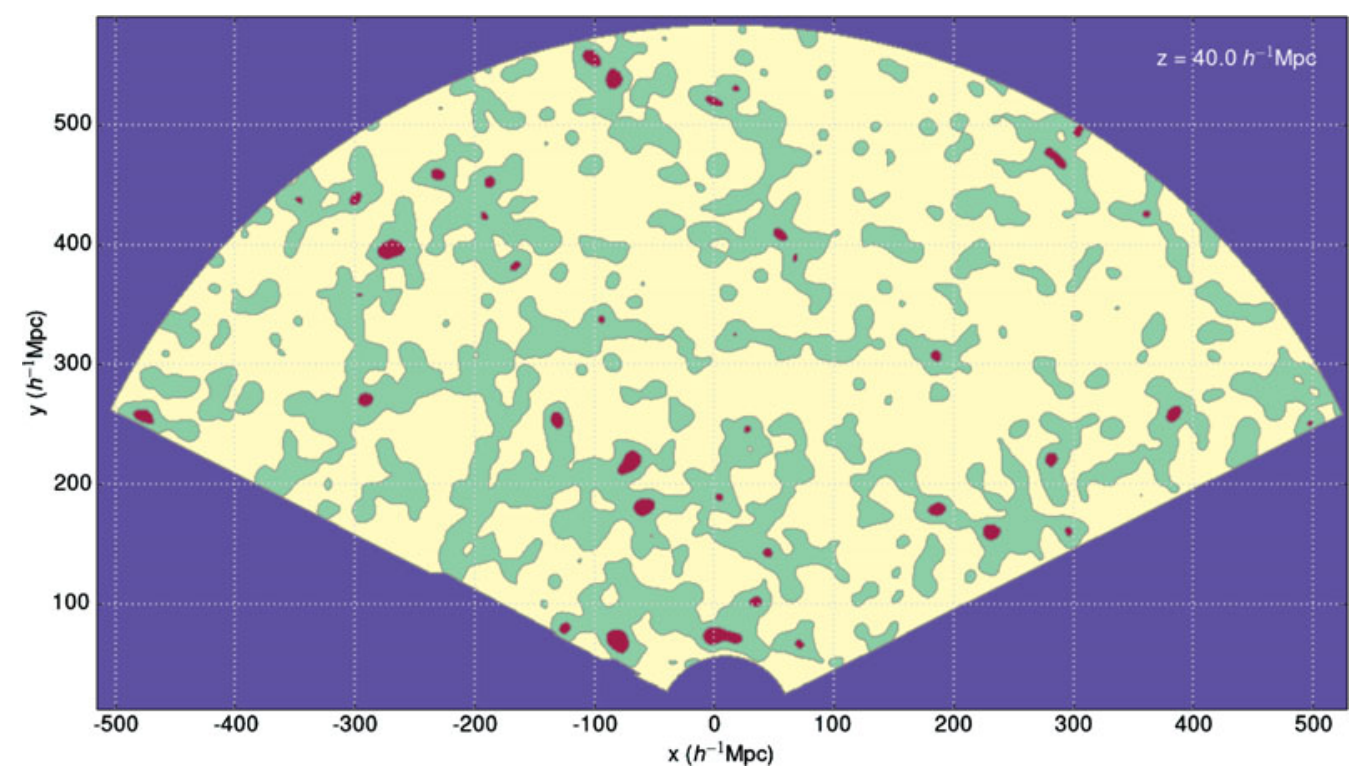

Figure 1. An illustrative slice of the SDSS luminosity density field, smoothed with $8 \mathrm{Mpc}$ B3 spline kernel. Void regions are marked with light gray (yellow), superclusters with dark gray (red) in the paper (electronic) version, respectively. The rest of the field has intermediate luminosity density. See text for the density level definitions.

and is complete down to $M_{r}=-18$ mag. Further on, the sample is split into different subsamples with correspondingly lower membership.

For characterising the environment, we have applied the large scale luminosity density, derived by smoothing with a B3 spline kernel within $8 \mathrm{Mpc}$ radius (Tempel et al. 2014). In addition to specifically probing the large-scale environment, the large smoothing radius minimises the contamination of the density field by the luminosity of the galaxy under consideration and its nearby neighbours. We compare galaxies within two extremes: void environment (density below the mean value of the Universe) and supercluster environment (density more than five times the mean value of the Universe). A slice of the SDSS luminosity density field with the corresponding density levels marked is shown in Fig. 1.

The morphological types of the galaxies (E or S) are taken from two independent sources: the visual classifications from the Galaxy Zoo Project (Lintott et al. 2008) and parameter-based determinations from (Tempel et al. 2011). A closer inspection reveals that both sources still contain obvious misclassifications, thus for security reasons, we have considered only those galaxies for which the two classifications overlap.

The photometric properties of the galaxies used in this study are taken from the SDSS. In addition, we use structural parameters from Simard et al. (2011), where bulge+disc decomposition of the SDSS galaxies was performed.

\section{Output}

Colour histograms of elliptical and spiral galaxies are shown in Fig. 2. As expected, the colour distribution of elliptical galaxies is relatively concentrated, with a single peak. The large-scale environment has only minor effects, narrowing the distribution slightly and shifting the maximum of $(g-i)$ by about 0.05 mag redward. On the other hand, the colour distribution of spirals is strongly bimodal in both environments. A clear density evolution is present: while blue spirals vastly dominates in void environmets, the numbers of 


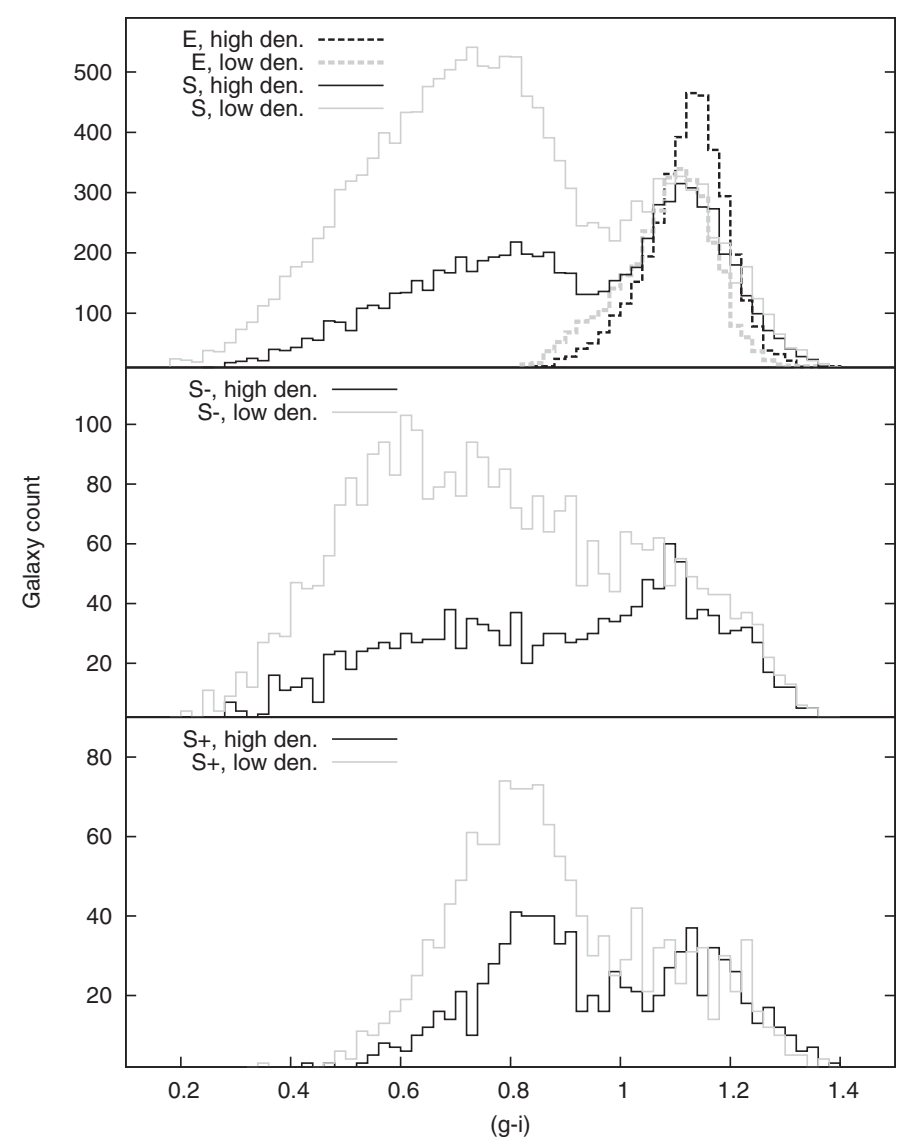

Figure 2. Colour histrogram of the volume-limited sample of SDSS galaxies in voids and in superclusters. Top panel - all ellipticals and spirals; middle panel - low luminosity spirals; bottom panel - high luminosity spirals. The strongest evolution with the large scale environment occurs for low luminosity spirals.

blue and red spirals become roughly balanced in supercluster environments. Apparently, the effect results from suppressed starformation, caused by the deficit of gas in denser environments. For investigating this effect further, we have split the spiral sample into lower $\left(r\right.$-band absolute luminosity $\left.L_{r}<0.5 \cdot 10^{10} h^{-2} L_{\odot}\right)$ and higher $\left(L_{r}>1 \cdot 10^{10} h^{-2} L_{\odot}\right)$ luminosity bins. A comparison of the middle and bottom panels of Fig. 2 shows that the loss of the blue peak is more pronounced for lower luminosity galaxies. In dense environments, low luminosity spirals achieve a nearly flat colour distribution, with the small red peak possibly caused by still inevitably present misclassifications.

Interesting aspects emerge also from correlating the structural parameters derived by Simard et al. (2011) with the environment density. For the spiral population of galaxies, the mean Sérsic index $n$, describing the shape of the surface brightness distribution, increases notably from 1.1 to 1.3. Galaxy-galaxy interactions and accretion of satellites are the potential culprits in this affair. A similar trend can be observed for low luminosity ellipticals (increase from 3.8 to 4 ), but the opposite takes place with luminous ellipticals (decrease from 3.5 in voids vs 3.0 in superclusters). One might suspect that the decrease of $n$ with environment density is related to the central ellipticals of groups and clusters, but after segregating ellipticals ranking first in luminosity within their groups and clusters 
from the others (according to Tempel et al. 2014), we find that the effect concerns both subsamples of luminous ellipticals.

\section{Conclusions}

The properties of both elliptical and spiral galaxies change with the large-scale environment density. Void spirals tend to be bluer and more compact than spirals in superclusters. Low- and high-luminosity ellipticals react differently to environment density increase: while both become slightly redder, the former become more centrally concentrated while the latter do not.

\section{Acknowledgements}

We acknowledge the financial support from the Estonian Research Council and the Centre of Excellence of Dark Matter in (Astro)particle Physics and Cosmology. This research is based on the SDSS datasets and the authors feel indebted to the SDSS team and their funding organisations; unfortunately, the page limit does not enable us to include the whole officially requested acknowledgment here.

\section{References}

Ahn, C. P., Alexandroff, R., Allende Prieto, C., et al. 2014, ApJ, 211, 17

Davis, M. \& Geller, M. J. 1976, ApJ, 208, 13

Dressler, A. 1980, ApJ, 236, 351

Einasto, J., Saar, E., Kaasik, A., \& Chernin, A. D. 1974, Nature, 252, 111

Einasto, M., Lietzen, H., Tempel, E., Gramann, M., Liivamägi, L. J., \& Einasto, J. 2014, A\&̛A, 562,87

Kauffmann, G., White, S. D. M., Heckman, T. M., Ménard, B., Brinchmann, et al., 2004, MNRAS, 353, 713

Lietzen, H., Tempel, E., Heinämäki, P., Nurmi, P., Einasto, M., \& Saar, E. 2012, A\&A, 545, $104 \mathrm{~L}$

Lintott, C. J., Schawinski, K., Slosar, A., et al. 2008, MNRAS, 389, 1179

Oemler, Jr., A. 1974, ApJ, 194, 1

Postman, M. \& Geller, M. J. 1984, ApJ, 281, 95

Simard, L., Mendel, J. T., Patton, D. R., Ellison, S. L., \& McConnachie, A. W. 2011, ApJS, 196,11

Tempel, E., Saar, E., Liivamägi, L. J., Tamm, A., Einasto, J., Einasto, M., \& Müller, V. 2011, $A \mathscr{E} A, 529,53$

Tempel, E., Tamm, A., Gramann, M., et al. 2014, A\&A, 566, A1

York, D. G., Adelman, J., Anderson, Jr., J. E., et al. 2000, AJ, 120, 1579 